\title{
Correction
}

\section{Correction: Kokkinaki et al., Klotho Regulates Retinal Pigment Epithelial Functions and Protects Against Oxidative Stress}

In the article "Klotho Regulates Retinal Pigment Epithelial Functions and Protects Against Oxidative Stress" by Maria Kokkinaki, Mones Abu-Asab, Nishantha Gunawardena, Gerard Ahern, Monica Javdnia, John Young, and Nady Golestaneh, which appeared on pages 16346-16359 of the October 9, 2013 issue, there was an error in Figure 3F. The original published figure of the Western blot was compiled with splicing out portions of the Western blot. This splicing was not evident in the image. The corrected figure below identifies the site of splicing in Figure $3 F$. This correction does not affect the results, interpretation, or conclusions reported in the article. Figure 3 has been corrected in the online PDF version and a corrected version displayed below.

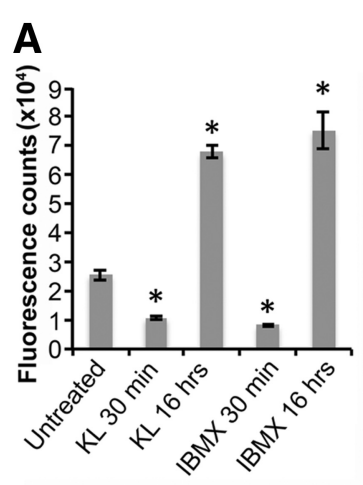

E

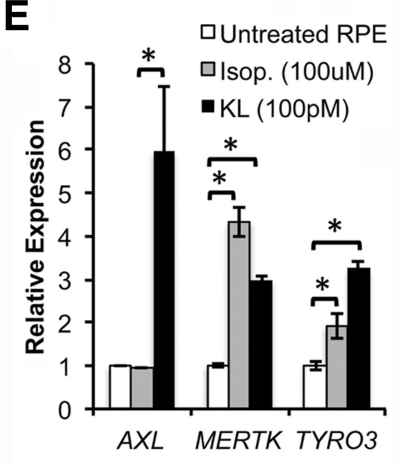

H

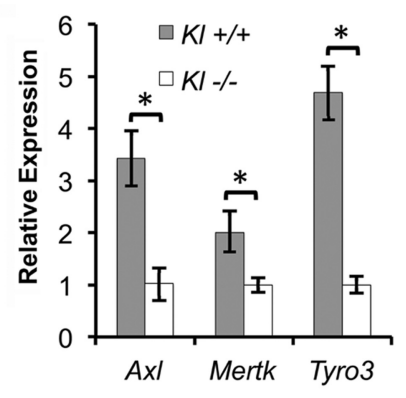

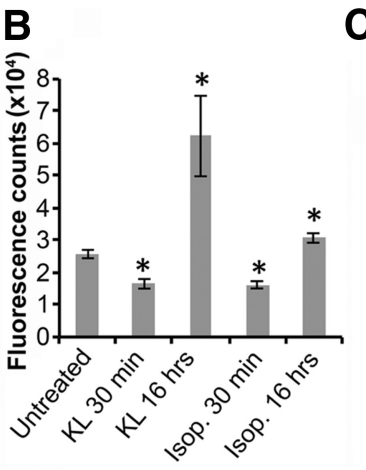

F

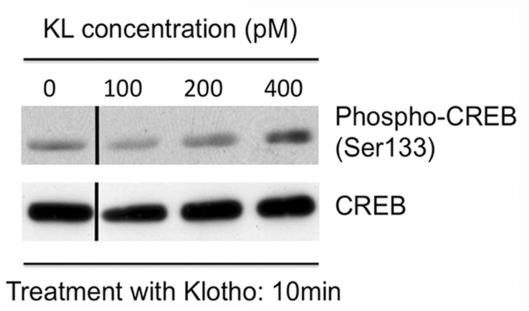

I

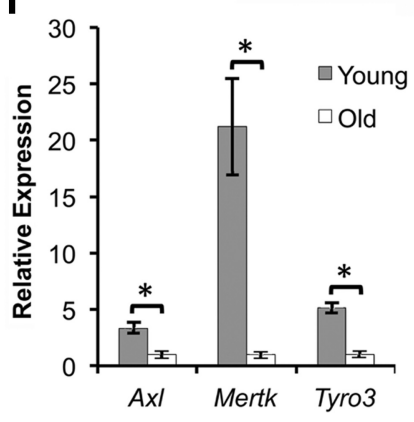

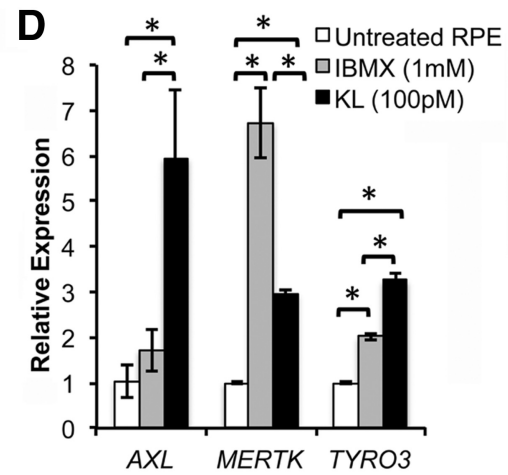

G

Time of treatment with 100pM KL

$0 \quad 5^{\prime} \quad 10^{\prime} \quad 15^{\prime} \quad 30^{\prime} \quad 1 \mathrm{hr}$

Phospho-Adducin (Ser481)

Adducin

Figure 3. 